\title{
GENERAL WIENER-HOPF OPERATORS AND THE NUMERICAL RANGE OF AN OPERATOR
}

\author{
VICTOR J. PELLEGRINI
}

\begin{abstract}
Let $H$ be a separable Hilbert space and $A$ a bounded operator on $H$. For a selfadjoint projection $P$ on $H$ we consider the general Wiener-Hopf operator $T_{P}(A)=P A_{R(P)}$ where $R(P)$ denotes the range of $P$. In this paper we study the relation between $T_{P}(A)$ and $W(A)$, the numerical range of $A$. In particular we characterize those operators $A$ such that $T_{P}(A)$ is invertible for every $P$.
\end{abstract}

We begin in $\S 1$ with a factorization theorem motivated by a classical result of Wiener and Hopf (see Introduction to [1] for a discussion). We use this in $\S 2$ to characterize those operators $A$ for which $T_{P}(A)$ is $1-1$ for every $P$. $\S 3$ contains the characterization of those operators $A$ such that $T_{P}(A)$ is invertible for every $A$. In $\S 4$ we strengthen the factorization result of $\S 1$ under the assumption that $A$ is normal and its numerical range lies in a cone.

Before proceeding with the main body of the paper the author wishes to thank Professor Marvin Shinbrot for suggestions which simplified the proof of Theorem 1.3.

1. Our aim in this section is to prove a factorization theorem similar to that in [1, Theorem 5]. We assume throughout this section that $A$ is a bounded, 1-1 operator on $H$ with range, $R(A)$, dense in $H$ and that $P$ is a fixed selfadjoint projection on $H$.

Let $\left\{\psi_{n}^{+}\right\},\left\{\psi_{n}^{-}\right\}$and $\left\{\phi_{n}^{+}\right\}$be complete orthonormal systems in $\operatorname{cl}\left(R\left(A^{*} P\right)\right), R(Q)$ and $R(P)$ respectively (where $\left.Q=I-P\right)$. Since $R(A)$ is dense, $A^{*}$ is $1-1$. Thus the cardinalities of $\left\{\psi_{n}^{+}\right\}$and $\left\{\phi_{n}^{+}\right\}$are the same.

1.1 Lemma. The formula $S f=\sum\left(f, \psi_{n}^{+}\right) \phi_{n}^{+}+\sum\left(f, \psi_{n}^{-}\right) \overline{\psi_{n}^{-}}$defines a bounded linear operator on $H$ whose adjoint is given by $S^{*} g=\sum\left(g, \phi_{n}^{+}\right) \psi_{n}^{+}+$ $\sum\left(g, \overline{\psi_{n}}\right) \psi_{n}^{-}$.

Received by the editors June 5, 1972 and, in revised form, July 12, 1972.

AMS (MOS) subject classifications (1970). Primary 47B35, 47A20; Secondary 47A10, 45E10.

Key words and phrases. General Wiener-Hopf operators, numerical range.

(C) American Mathematical Society 1973 
Proof. Let $T$ be the orthogonal projection on $\operatorname{cl}\left(R\left(A^{*} P\right)\right)$. Then for each $f \in H$

$$
\begin{aligned}
\|S f\|^{2} & =\sum\left|\left(f, \psi_{n}^{+}\right)\right|^{2}+\sum\left|\left(f, \psi_{n}^{-}\right)\right|^{2} \\
& \leqq\|T f\|^{2}+\|Q f\|^{2} \\
& \leqq 2\|f\|^{2} .
\end{aligned}
$$

Thus $S$ is bounded. The formula for $S^{*}$ is a straightforward computation.

1.2 Lemma. If $T_{P}(A)$ is $1-1$ then $S$ is $1-1$.

Proof. The formula for $S^{*}$ yields $S^{*} \phi_{k}^{+}=\psi_{k}^{+}$and $S^{*} \psi_{k}^{-}=\psi_{k}^{-}$where $\phi_{k}^{ \pm}$and $\psi_{k}^{t}$ are as in 1.1. From this we conclude that $\operatorname{cl}\left(R\left(S^{*}\right)\right)$ contains the space $\operatorname{cl}\left(R\left(A^{*} P\right)\right)+R(Q)$. If $h$ is orthogonal to $\operatorname{cl}\left(R\left(S^{*}\right)\right)$, then it is orthogonal to $R(Q)$ and thus $h \in R(P)$. Since $h \in R(P)$ and $h$ is orthogonal to $\operatorname{cl}\left(R\left(A^{*} P\right)\right)$, we have that $\left(T_{P}\left(A^{*}\right) p, h\right)=\left(A^{*} p, h\right)=0$ for each $p \in R(P)$. Thus $h \in \operatorname{cl}\left(R\left(T_{P}\left(A^{*}\right)\right)\right)^{\perp}=N\left(T_{P}(A)\right)=\{0\}$, where $N\left(T_{P^{2}}(A)\right)$ denotes the null space $T_{P^{\prime}}(A)$. Thus $\operatorname{cl}\left(R\left(S^{*}\right)\right)^{\perp}=N(S)=\{0\}$.

1.3 TheOREM. Let $A$ be a bounded, 1-1 operator on $H$ whose range, $R(A)$, is dense in $H$. Let $P$ be a selfadjoint projection on $H$. Then if $T_{P}(A)$ is 1-1, we may factor $A$ as $A=A_{-} A_{+}$where

(1) $A_{+}$is a bounded, 1-1 operator satisfying $R\left(A_{+} P\right) \subset R(P)$,

(2) $A_{-}$is a 1-1 operator with $D\left(A_{-}\right)=R\left(A_{+}\right)$satisfying $R\left(A_{-}^{-1} Q\right) \subset R(Q)$.

Proof. Since $T_{P}(A)$ is $1-1$, we have by 1.3 that $S$ is $1-1$. Let $A_{+}=S$ and $A_{-}=A S^{-1}$.

If $p \in R(P)$, then $S p=\sum\left(p, \psi_{n}^{+}\right) \phi_{n}^{+}+\sum\left(p, \psi_{n}^{-}\right) \psi_{n}^{-}=\Sigma\left(p, \psi_{n}^{+}\right) \phi_{n}^{+}$. Thus $S p \in R(P)$.

If $q \in R(Q) \cap D\left(A^{-1}\right)$, we have $\left(A^{-1} q, \psi_{n}^{+}\right)=0$ for all $n$ since $\psi_{n}^{+} \in$ $\operatorname{cl}\left(R\left(A^{*} P\right)\right)$. Thus for $q \in R(Q) \cap D\left(A_{-}^{-1}\right)=R(Q) \cap D\left(A^{-1}\right)$ we have

$$
A_{-}^{-1} q=\sum\left(A^{-1} q, \psi_{n}^{+}\right) \phi_{n}^{+}+\sum\left(A^{-1} q, \psi_{n}^{-}\right) \psi_{n}^{-}=\sum\left(A^{-1} q, \psi_{n}^{-}\right) \psi_{n}^{-}
$$

which implies $A_{-}^{-1} q \in R(Q)$.

1.4 Corollary. If $T_{P}(A) u=v$ where $v \in R(P) \cap D\left(A^{-1}\right)$, then $u=$ $A_{+}^{-1} P A_{-}^{-1} v$.

Proof. We have $A u=v+q$ where $q \in R(Q)$. Thus $A_{+} u=A_{-}^{-1} v+A_{-}^{-1} q$. Hence $A_{+} u=P A_{-}^{-1} v$, from which the result follows.

2. We now characterize those bounded operators $A$ with the property that $T_{P},(A)$ is $1-1$ for every orthogonal projection $P$. We introduce some notation. A factorization of the type given in 1.3 will be called a WienerHopf factorization ( $W-H$ factorization) for $A$ with respect to $P$. If the factors $A_{-}$and $A_{+}$are invertible and satisfy $R\left(A_{+} P\right)=R(P)$ and 
$R\left(A_{-}^{-1} Q\right)=R(Q)$, we call the factorization a strong Wiener-Hopf factorization.

2.1 THEOREM. Let $A$ be a bounded operator on $H$. Then the following are equivalent:

(1) $T_{P}(A)$ is 1-1 for every projection $P$,

(2) A admits a $W$ - $H$ factorization with respect to every projection $P$,

(3) There exists a complex number $\lambda_{0}$ of absolute value 1 such that $\operatorname{Re} \lambda_{0} A>0$.

Proof. Suppose that (1) holds. Let $W(A)=\{(A x, x):\|x\|=1\}$ be the numerical range of $A$. Suppose $0 \in W(A)$. Then there exists an $x_{0} \neq 0 \ni$ : $\left(A x_{0}, x_{0}\right)=0$. Let $P_{0}$ be the projection on the one dimensional subspace spanned by $x_{0}$. Thus $P_{0} y=\left(y, x_{0}\right) x_{0}$ for each $y \in H$. Then $T_{I_{0}}(A) x_{0}=0$ which is a contradiction. Since $0 \notin W(A)$ and $W(A)$ is convex [3, Problem $166]$ we conclude that (3) holds.

Now suppose that (3) holds. Let $P$ be a projection. Then the operator $\lambda_{0} A$ satisfies the conditions of 1.3 and thus admits a $W-H$ factorization with respect to $P, \lambda_{0} A=C_{-} C_{+}$. Hence $A=\left(\lambda_{0}^{-1} C_{-}\right)\left(C_{+}\right)$is the required factorization of $A$ with respect to $P$. Hence (2) holds.

Finally, suppose (2) holds. Let $P$ be a projection and $p \in R(P)$. If $T_{P}(A) p=0$ then $A_{-} A_{+} p=q$ where $q \in R(Q) \quad(Q=I-P)$. This implies $A_{+} p=A_{-}^{-1} q$ and thus that $A_{+} p \in R(P) \cap R(Q)$. Hence $A_{+} p=0$ and, since $A_{+}$is $1-1, p=0$.

3. We are now ready to present our main result. Before doing so let us review some pertinent results of Devinatz and Shinbrot. In [1] they showed that for a fixed projection $P$ the following conditions are equivalent for an invertible operator $A$ :

(i) $T_{P}(A)$ is invertible,

(ii) $A$ admits a strong $W-H$ factorization with respect to $P$,

(iii) There exists an invertible operator $L$ and $\delta>0$ such that $\operatorname{Re} A L \geqq$ $\delta>0$ and $R(L P)=R(P)$.

The operator $L$ depends on the particular choice of $P$. If $T_{P}(A)$ is invertible for all $P$ we will show that one can find an operator $L$ which works for all $P$. More precisely we have

3.1 THEOREM. Let $A$ be a bounded operator on $H$. Then the following are equivalent:

(i) $T_{P^{\prime}}(A)$ is invertible for every $P$,

(ii) $A$ admits a strong $W$ - $H$ factorization with respect to every $P$,

(iii) There exists a complex number $\lambda_{0}$ of absolute value 1 and a real number $\delta>0$ such that $\operatorname{Re} \lambda_{0} A \geqq \delta$. 
Proof. (i) is equivalent to (ii) by the result of Devinatz and Shinbrot stated above.

Suppose (iii) holds. Then $0 \notin \operatorname{cl}\left(W\left(\lambda_{0} A\right)\right)$. Since $\operatorname{cl}\left(W\left(T_{P}\left(\lambda_{0} A\right)\right)\right)$ contains the spectrum of $T_{1},\left(\lambda_{0} A\right)$ [3, Problem 169] and lies in $\operatorname{cl}\left(W\left(\lambda_{0} A\right)\right)$, we conclude that $T_{P}\left(\lambda_{0} A\right)$ is invertible for each $P$. Thus $T_{P}(A)$ is invertible for each $P$. Hence (i) holds.

It remains to show that (i) implies (iii). Since $T_{P}(A)$ is invertible for every projection $P$ it follows by 2.1 that there exists a $\lambda_{0}$ of absolute value 1 such that $\operatorname{Re} \lambda_{0} A>0$. Suppose that $0 \in \operatorname{cl}(W(A))$. Then there exists a sequence $\left\{x_{n}\right\}$ of unit vectors such that $\left(A x_{n}, x_{n}\right) \rightarrow 0$. Since the unit ball of $H$ is compact and metrizable in the weak topology the sequence $\left\{x_{n}\right\}$ has a weakly convergent subsequence $\left\{x_{n_{k}}\right\}$. Let $y=\lim _{k \rightarrow \infty} x_{n_{k}}$. By the generalized Schwartz inequality we have

$$
\begin{aligned}
\left(\operatorname{Re} \lambda_{0} A y, y\right)^{2} & =\lim \left|\left(\operatorname{Re} \lambda_{0} A y, x_{n_{k}}\right)\right|^{2} \\
& \leqq \lim \left(\operatorname{Re} \lambda_{0} A y, y\right)\left(\operatorname{Re} \lambda_{0} A x_{n_{k}}, x_{n_{k}}\right) \\
& =0
\end{aligned}
$$

(since $\left(A x_{n}, x_{n}\right) \rightarrow 0$ implies $\left.\left(\operatorname{Re} \lambda_{0} A x_{n_{k}}, x_{n_{k}}\right) \rightarrow 0\right)$. Thus, since $\lambda_{0} A>0$, we conclude that $y=0$. Thus there exists a sequence $\left\{x_{n_{k}}\right\}$ of unit vectors such that $\left(A x_{n_{k}}, x_{n_{k}}\right) \rightarrow 0$ and $x_{n_{k}} \rightarrow 0$ weakly. Hence by [2, Theorem 5.1], there exists an infinite dimensional projection $P_{0} \ni: T_{P_{0}}(A)$ is compact. Hence $T_{P_{0}}(A)$ is not invertible. This is a contradiction. Thus $0 \notin \operatorname{cl}(W(A))$. Since $\operatorname{cl}(W(A))$ is a closed convex set (iii) follows.

3.2 Corollary. $T_{P}(A)$ is invertible for every $P$ if and only if $0 \notin$ $\operatorname{cl}(W(A))$.

For an operator $B$ let $\sigma(B)$ denote its spectrum.

3.3 Corollary. $\lambda \notin \sigma\left(T_{P}(A)\right)$ for every $P$ if and only if $\lambda \notin \operatorname{cl}(W(A))$. Thus $\mathrm{cl}(W(A))=\bigcup\left\{\sigma\left(T_{P}(A)\right): P\right.$ a selfadjoint projection $\}$.

Remarks. (1) Let $A$ be a bounded operator on a Hilbert space $H$. A complex number $\lambda$ is said to be in the essential numerical range of $A$ if there exists a sequence of unit vectors $\left\{x_{n}\right\}$ such that $\left(A x_{n}, x_{n}\right) \rightarrow \lambda$ and $x_{n} \rightarrow 0$ weakly [2]. We have shown, in the proof of 3.1 that $\operatorname{cl}(W(A)) \backslash W(A)$, where the slash signifies complement, lies in the essential numerical range of $A$.

(2) If $A$ is not a nonzero scalar multiple of the identity then Williams has shown in [5, Theorem 3] that there exists an invertible operator $S$ on $H$ such that $0 \in W\left(S^{-1} A S\right)$. Hence by 3.1 (or by 2.1 ) there exists a selfadjoint projection $P \ni: T_{P}\left(S^{-1} A S\right)$ is not invertible. If we let $E=S P S^{-1}$ then $E$ is a skew projection (i.e. $E^{2}=E$ but not necessarily selfadjoint) such that 
$E A E \mid R(E)$ is not invertible. From this we conclude that $E A E \mid R(E)$ is invertible for every skew projection $E$ if and only if $A$ is a nonzero scalar multiple of the identity.

4. The factorization in $\S 1$ obtained under the assumption $0 \notin W(A)$ is considerably weaker than the one obtained by Devinatz and Shinbrot under the assumption $0 \notin \operatorname{cl}(W(A))$. In this section we show that the assumption of normality and a further restriction on $W(A)$ (short of $0 \notin$ $\operatorname{cl}(W(A)))$ leads to a strengthening of the factorization in $\S 1$. The conditions imposed on $A$ are (except for the assumption that $A$ is bounded) a natural generalization of those in [4].

The following lemma can be proved by a simple adaptation of the techniques used in the proof of [1, Theorem 5].

4.1 Lemma. Let $N$ and $M$ be closed subspaces of a Hilbert space $H$ such that $\operatorname{dim} N=\operatorname{dim} M$ and $\operatorname{dim} N^{\perp}=\operatorname{dim} M^{\perp}$. Let $V$ be a bounded operator on $H$ satisfying $\operatorname{Re} V \geqq \delta>0$. Then there exist invertible operators $V_{-}$and $V_{+}$on $H$ such that

(1) $V=V_{-} V_{+}$,

(2) $V_{+} M=N, V_{-} N^{\perp}=M^{\perp}$.

4.2 Lemma. Let $V$ and $B$ be bounded operators on $H$. Suppose $V$ is as in 4.1, and $B>0$ and $V$ commutes with $B$. If $A=V \cdot B$, then there exists bounded, 1-1 operators $A_{-}$and $A_{+}$on $H$ such that

(1) $A=A_{-} A_{+}$,

(2) $\operatorname{cl}\left(R\left(A_{+} P\right)\right)=R(P), R\left(A_{-} Q\right)=R(Q)$.

Proof. Let $M=\operatorname{cl}\left(R\left(B^{1 / 2} P\right)\right)$ and $N=R(P)$. Then $M^{\perp}=R\left(B^{-1 / 2} Q\right)$ and $N^{\perp}=R(Q)$. Applying 4.1 to $V$ we have $V=V_{-} V_{+}$where $V_{+} \operatorname{cl}\left(R\left(B^{1 / 2} P\right)\right)=$ $\operatorname{cl}\left(R\left(V_{+} B^{1 / 2} P\right)\right)=R(P)$ and $V_{-} R(Q)=R\left(B^{-1 / 2} Q\right)$. Set $A_{+}=V_{+} B^{1 / 2}$ and $A_{-}=B^{1 / 2} V_{-}$. Then $A_{-} A_{+}=B^{1 / 2} V_{-} V_{+} B^{1 / 2}=B^{1 / 2} V B^{1 / 2}=B V=A$.

Definition. Let $C>0$ and $z=x+i y$. The set $\{z:|y| \leqq c x\}$, or any rotation of such a set, will be called a cone.

For the remainder of this paper we will assume that $A$ is $1-1$ and normal. Thus we may write $A=V B$ where $V$ is unitary, $B>0$, and $V$ commutes with $B$.

4.3 Lemma. Let $A$ be a normal, $1-1$ operator. Let $K_{+}$be a cone lying in the right half-plane. Suppose $W(A) \subset K_{+}$. Then there exists a $\delta>0$ such that $\operatorname{Re} V \geqq \delta>0$.

Proof. $W(A) \subset K_{+}$implies $\{(A x, x): x \in H\} \subset K_{+}$. Let $\phi_{V}: H \rightarrow C$ be defined by $\phi_{V}(x)=(V x, x)$. Then $\phi_{V}\left(B^{1 / 2} x\right)=\left(V B^{1 / 2} x, B^{1 / 2} x\right)=(A x, x) \in$ $K_{+}$since $B^{1 / 2}$ commutes with $V$. Since $\phi_{V}$ is continuous, $K_{+}$is closed and 
$R\left(B^{1 / 2}\right)$ is dense in $H$ we conclude that $\{(V x, x): x \in H\} \subset K_{+}$. Thus $\sigma(V) \subset$ $\operatorname{cl}(W(V)) \subset K_{+}$. This implies $\sigma(V) \subset\{z:|z|=1\} \cap K_{+}$and hence co $\sigma(V)$, the closed convex hull of $\sigma(V)$, is contained in a set of the form $\{z: \operatorname{Re} z \geqq \delta\}$. Finally since $V$ is normal, co $\sigma(V)=\operatorname{cl}(W(V))$ [3, Problem 171], and we have our result.

4.4 THEOREM. Let A be a 1-1 normal operator whose numerical range is contained in a cone. Let $P$ be a selfadjoint projection and $Q=I-P$. Then there exist bounded, 1-1 operators having dense ranges such that

(1) $A=A_{-} A_{+}$,

(2) $\operatorname{cl}\left(R\left(A_{+} P\right)\right)=R(P), R\left(A_{-} Q\right)=R(Q)$.

Proof. There exists a $\phi_{0}, 0 \leqq \phi_{0}<2 \pi$, such that $e^{i \phi_{0}} A$ satisfies the conditions of 4.3.

We conclude by remarking that a version of 4.4 is valid when $A$ is unbounded. Details will appear in a later paper.

\section{REFERENCES}

1. A. Devinatz and M. Shinbrot, General Wiener-Hopfoperators, Trans. Amer. Math. Soc. 145 (1969), 467-494. MR 40 \#4800.

2. P. A. Fillmore, J. G. Stampfli and J. P. Williams, On the essential numerical range, the essential spectrum, and a problem of Halmos (to appear).

3. P. R. Halmos, A Hilbert space problem book, Van Nostrand, Princeton, N.J., 1967. MR 34 \#8178.

4. M. Shinbrot, On the range of general Wiener-Hopf operators, J. Math. Mech. 18 (1968/69), 587-601. MR 40 \#3375.

5. J. P. Williams, Similarity and the numerical range, J. Math. Anal. Appl. 26 (1969), 307-314. MR 39 \#2010.

Department of Mathematics, University of Cincinnati, Cincinnati, Ohio 45221 